\title{
PARAMETRIC VIBRATION OF THE PRISMATIC SHAFT WITH HEREDITARY AND NONLINEAR GEOMETRY
}

\author{
HOANG VAN DA \\ Hanoi Technical University of Geology and Mining \\ INTRODUCTION
}

Parametric vibration of the prismatic shaft with regard of physical and geometrical nonlinearity has been investigated in some publications (see for example $[1,2,4,5]$. However, that vibration in the case of hereditary has not, to author's knowledge, been examined hitherto. In this paper it will be studied by means of the asymptotic method for high order systems.

\section{FORMULATION OF THE PROBLEM. THE EQUATION OF MOTION}

Let us study parametric vibration of shaft of the length $\ell$ supported in horizontal position as shown in fig. 1 and acted on the longitudinal periodic force. Supposing that the nondeformed axis of the shaft coincides with axis $O x$, while the symmetric axes of the cross-section are parallel to the fixed axes $O z$ and $O y$. In addition the origin of the coordinates is selected on the shaft's left end, see fig. 1.

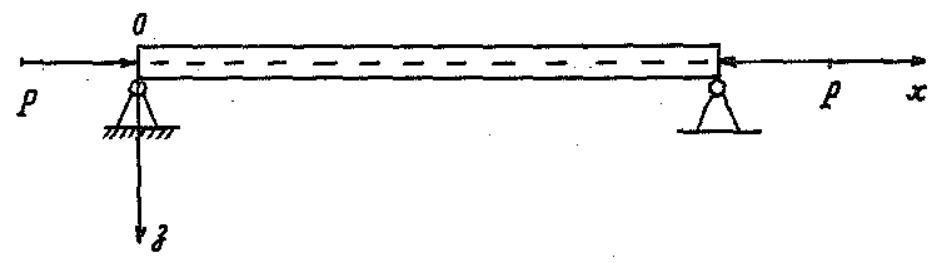

Fig. 1

Transferences at points of the axis $O x$ in the direction of axes $O z, O x$ are expressed by functions $W(x, t), U(t)$. Neglecting the inertia of rotatory motion and the displacement, the equations of the considered boundary value problem are

$$
\begin{aligned}
\frac{\partial N}{\partial x}-\frac{\partial}{\partial x}\left(Q \frac{\partial W}{\partial x}\right)-\rho F \frac{\partial^{2} U}{\partial t^{2}} & =0 \\
\frac{\partial^{2} M}{\partial x^{2}}+\frac{\partial}{\partial x}\left(N \frac{\partial W}{\partial x}\right)-\rho F \frac{\partial^{2} W}{\partial t^{2}} & =0
\end{aligned}
$$

Here $M(x, t)$ is bending moment of cross-section, $F$ - its area, $\rho$ - specific mass, $N$ - normal force, $Q$ - cross force.

The equation of state is accepted in [11]

$$
\sigma=a_{1} \varepsilon_{x}+a_{2} \varepsilon_{x}^{3}+\int_{0}^{t} K(t-\tau) \frac{\partial}{\partial \tau}\left(\varepsilon_{x}\right) d \tau
$$


where $a_{1}, a_{2}$ are the constant characteristing the properties of material [10], $K(t-\tau)$ - function of hereditary,

$$
\varepsilon_{x}=\frac{\partial U}{\partial x}+\frac{1}{2}\left(\frac{\partial W}{\partial x}\right)^{2}-z \frac{\partial^{2} W}{\partial x^{2}} .
$$

Bending moment $M$ and normal force $N$ are determined by the following expressions

$$
\begin{aligned}
& M= \iint_{F} \sigma z d F, \\
& M=-a_{1} I_{0} \frac{\partial^{2} W}{\partial x^{2}}-a_{2} I_{2}\left(\frac{\partial^{2} W}{\partial x^{2}}\right)^{3}-3 a_{2} I_{0} \varepsilon_{0}^{2} \frac{\partial^{2} W}{\partial x^{2}}-I_{0} \int_{0}^{t} K(t-\tau) \frac{\partial^{3} W}{\partial \tau \partial x^{2}} d \tau, \\
& \quad N=\iint_{F} \sigma d F, \\
& \quad N=a_{1} F \varepsilon_{0}+a_{2} F \varepsilon_{0}^{3}+3 a_{2} I_{0} \varepsilon_{0}\left(\frac{\partial^{2} W}{\partial x^{2}}\right)^{2}+F \int_{0}^{t} K(t-\tau) \frac{\partial \varepsilon_{0}}{\partial t} d \tau .
\end{aligned}
$$

Here $I_{0}, I_{2}$ are the cross-section moments of inertia

$$
I_{0}=\iint_{F} z^{2} d F, \quad I_{2}=\iint_{F} z^{4} d F
$$

$\varepsilon_{0}$ is the lengthener of the shaft's axis $[10]$

$$
\varepsilon_{0}=\frac{\partial U}{\partial x}+\frac{1}{2}\left(\frac{\partial W}{\partial x}\right)^{2} .
$$

Neglecting the longitudinal inertial force $\rho F \frac{\partial^{2} U}{\partial t^{2}}$ and influence of the cross force $Q$, we have from the equation (1.1) and (1.6) the expression $N=N(t)$

$$
a_{1} F \varepsilon_{0}+a_{2} F \varepsilon_{0}^{3}+\left.3 a_{2} I_{0} \varepsilon_{0}\left(\frac{\partial^{2} W}{\partial x^{2}}\right)^{2}\right|_{x=0, \ell}+F \int_{0}^{t} K(t-\tau) \frac{\partial \varepsilon_{0}}{\partial \tau} d \tau=-P(t)
$$

Supposing that the nonlinear terms in (1.7) are small enough and applying the successive approximate method [12] we get

$$
\varepsilon_{0}=-\frac{P}{a_{1} F}+\frac{a_{2}}{a_{1}^{4}} \frac{P^{3}}{F^{3}}+\left.\frac{3 a_{2} I_{0}}{a_{1}^{2} F^{2}} P\left(\frac{\partial^{2} W}{\partial x^{2}}\right)^{2}\right|_{x=\ell}+\frac{1}{a_{1}^{2} F} \int_{0}^{t} K(t-\tau) \frac{d P}{d \tau} d \tau .
$$

By substuting (1.8) into the expression (1.5) and then into (1.2), after simple calculations we obtain the equation of motion of the boundary value problem

$$
\begin{aligned}
\frac{\partial^{2} W}{\partial t^{2}}+\frac{a_{1} I_{0}}{\rho F} \frac{\partial^{4} W}{\partial x^{4}}= & -\frac{3 a_{2} I_{2}}{\rho F}\left[\frac{\partial^{4} W}{\partial x^{4}}\left(\frac{\partial^{2} W}{\partial x^{2}}\right)^{2}+2\left(\frac{\partial^{2} W}{\partial x^{2}}\right)^{2} \frac{\partial^{2} W}{\partial x^{2}}\right]-\frac{I_{0}}{\rho F} \int_{0}^{t} K(t-\tau) \frac{\partial^{5} W}{\partial x^{4} \partial \tau} d \tau- \\
& -\frac{P}{\rho F} \frac{\partial^{2} W}{\partial x^{2}}-\frac{3 a_{2} I_{0}}{\rho F}\left[-\frac{P}{a_{1} F}+\frac{a_{2} P^{3}}{a_{1}^{4} F^{3}}+\left.\frac{3 a_{2} I_{0} P}{a_{1}^{2} F^{2}}\left(\frac{\partial^{2} W}{\partial x^{2}}\right)^{2}\right|_{x=\ell}+\right. \\
& \left.+\frac{1}{a^{2} F} \int_{0}^{t} K(t-\tau) \frac{d P}{d \tau} d \tau\right]^{2} \frac{\partial^{4} W}{\partial x^{4}}
\end{aligned}
$$


The boundary coxditions are approximately of the form

$$
\left.W\right|_{x=0}=0,\left.\quad \frac{\partial^{2} W}{\partial x^{2}}\right|_{x=0}=0,\left.\quad W\right|_{x=\ell}=0,\left.\quad \frac{\partial^{2} W}{\partial x^{2}}\right|_{x=\ell}=0 .
$$

\section{CONSTRUCTION OF SOLUTION}

We shall find the solution of the given boundary value problem by the help of the asymptotic method. In the first approximation the partial solution of the equation (1.9) with the boundary conditions (1.10) is found in the following forms:

$$
W(x, t)=y(t) \sin \frac{\pi x}{\ell}
$$

Puting (2.1) into (1.9) and applying Galerkin-Bubnovs method, we receive

$$
\begin{aligned}
\frac{d^{2} y}{d t^{2}}+\omega^{2} y= & \beta_{1} y^{3}+\beta_{2} \int_{0}^{t} K(t-\tau) \frac{d y}{d \tau} d \tau+\beta_{3} P y+ \\
& \beta_{4}\left[-\frac{P}{a_{1} F}+\frac{a_{2} P^{3}}{a_{1}^{4} F^{3}}+\frac{1}{a_{1}^{2} F} \int_{0}^{t} K(t-\tau) \frac{d P}{d \tau} d \tau\right]^{2} y
\end{aligned}
$$

where

$$
\omega^{2}=\frac{a_{1} I_{0} \pi^{4}}{\rho F \ell^{4}}, \quad \beta_{1}=-\frac{3 a_{2} I_{2} \pi^{8}}{4 \rho F \ell^{8}}, \quad \beta_{2}=-\frac{I_{0} \pi^{4}}{\rho F \ell^{4}}, \quad \beta_{3}=\frac{\pi^{2}}{\rho F \ell^{2}}, \quad \beta_{4}=-\frac{3 a_{2} I_{0} \pi^{4}}{\rho F \ell^{4}} .
$$

It is noted worthy that the term $\frac{\partial^{2} W}{\partial x^{2}}$ in the equation (1.9) vanishes when the coordinate $x$ is equal to $\ell$ or zero.

It is supposed that the function $K(t-r)$ and the force $P(t)$ are of the following forms

$$
\begin{aligned}
K(t-\tau) & =Q_{0} e^{-\alpha(t-\tau)}, \\
P(t) & =P_{0} \sin \gamma t
\end{aligned}
$$

here $Q_{0}, P_{0}$, a are positive constants Putting the expressions (2.3), (2.4) into equation (2.2) we have

$$
\begin{aligned}
\frac{d^{2} y}{d t^{2}}+\omega^{2} y= & \beta_{1} y^{3}+\beta_{2} Q_{0} \int_{0}^{t} e^{-\alpha(t-\tau)} \frac{d y}{d \tau} d \tau+\beta_{3} P_{0} \sin \gamma t y+ \\
& +\beta_{4}\left[-\frac{P_{0}}{a_{1} F} \sin \gamma t+\frac{a_{2} P_{0}^{3}}{a_{1}^{4} F^{3}} \sin ^{3} \gamma t+\frac{Q_{0} P_{0} \gamma}{a_{1} F} \int_{0}^{t} e^{-\alpha(t-\tau)} \cos \gamma \tau d \tau\right]^{2} y .
\end{aligned}
$$

Differentiating the equation (2.5) with respect to argument $t$ we get affter simple calculations the differential equation of third order

$$
\begin{aligned}
& \frac{d^{3} y}{d t^{3}}+\alpha \frac{d^{2} y}{d t^{2}}+\omega^{2} \frac{d y}{d t}+\alpha \omega^{2} y=\alpha \beta_{1} y^{3}+3 \beta_{1} y^{2} \frac{d y}{d t}+\left\{\beta_{3}\left[\alpha P_{0} \sin \gamma t+P_{0} \gamma \cos \gamma t\right]+\right. \\
& +\beta_{4}\left[\alpha C_{0}\left(\alpha C_{2}+2 \gamma b_{2}\right) \cos 2 \gamma t+\left(\alpha b_{2}-2 \gamma C_{2}\right) \sin 2 \gamma t+\left(\alpha C_{4}+4 \gamma b_{4}\right) \cos 4 \gamma t+\right. \\
& \left.\left.+\left(\alpha b_{4}-4 \gamma C_{4}\right) \sin 4 \gamma t+\alpha C_{6} \cos 6 \gamma t-6 C_{6} \gamma \sin 6 \gamma t\right]\right\} y+\left\{\beta_{2} Q_{0}+\beta_{3} P_{0} \sin \gamma t+\right. \\
& \left.+\beta_{4}\left[C_{0}+C_{2} \cos 2 \gamma t+b_{2} \sin 2 \gamma t+C_{4} \cos 4 \gamma t+b_{4} \sin 4 \gamma t+C_{6} \cos 6 \gamma t\right]\right\} \frac{d y}{d t},
\end{aligned}
$$


where

$$
\begin{gathered}
C_{0}=\frac{P_{0}}{2 a_{1}^{2} F}+\frac{P_{0} \gamma^{2}}{2 a_{1}^{4} F^{4}\left(\alpha^{2}+\gamma^{2}\right)}+\frac{3 a_{2} P_{0}^{4} \gamma^{2}}{4 a_{1}^{6} F^{4}\left(\alpha^{2}+\gamma^{2}\right)}+\frac{5 a_{2}^{2} P_{0}^{6}}{16 a_{1}^{8} F^{8}}-\frac{P_{0}^{2} \gamma^{2}}{a_{1}^{8} F^{2}\left(\alpha^{2}+\gamma^{2}\right)}-\frac{3 a_{2} P_{0}^{4}}{4 a_{1}^{5} F^{4}}, \\
C_{2}=\frac{P_{0}^{2} \gamma^{2}}{a_{1}^{3} F^{2}\left(\alpha^{2}+\gamma^{2}\right)}+\frac{P_{0}^{2} \gamma^{2}\left(\alpha^{2}-\gamma^{2}\right)}{2 a_{1}^{4} F^{2}\left(\alpha^{2}+\gamma^{2}\right)^{2}}+\frac{a_{2} P_{0}^{4}}{a_{1}^{5} F^{4}}-\frac{P_{0}^{2}}{2 a_{1}^{2} F^{2}}-\frac{15 a_{2}^{2} P_{0}^{6}}{32 a_{1}^{8} F^{6}}-\frac{a_{2} P_{0}^{4} \gamma^{2}}{a_{1}^{6} F^{4}\left(\alpha^{2}+\gamma^{2}\right)}, \\
C_{4}=-\frac{a_{2} P_{0}^{4} \gamma^{2}}{4 a_{1}^{6}\left(\alpha^{2}+\gamma^{2}\right)}+\frac{3 a_{2}^{2} P_{0}^{6}}{16 a_{1}^{8} F^{6}}-\frac{2 a_{2} P_{0}^{4}}{a_{1}^{5} F^{4}} \\
C_{6}=-\frac{a_{2}^{2} P_{0}^{6}}{32 a_{1}^{8} F^{6}}, \\
b_{2}=\frac{4 P_{0}^{2} \gamma^{3} \alpha}{a_{1}^{4} F\left(\alpha^{2}+\gamma^{2}\right)^{2}}+\frac{a_{2} P_{0}^{4} \gamma \alpha}{2 a_{1}^{6} F^{4}\left(\alpha^{2}+\gamma^{2}\right)}-\frac{4 P_{0}^{2} \alpha \gamma}{a_{1}^{3} F^{2}\left(\alpha^{2}+\gamma^{2}\right)}, \\
b_{4}=-\frac{a_{2} P_{0}^{4} \alpha \gamma}{a_{1}^{6} F^{4}\left(\alpha^{2}+\gamma^{2}\right)} .
\end{gathered}
$$

This equation will be solved by the asymptotic method [2]. Let's consider the case, when $\beta_{1}$, $\beta_{2}, \beta_{3}, \beta_{4}$ are small quantities of first order so that

$$
\beta_{1}=\varepsilon \bar{\beta}_{1}, \quad \beta_{2}=\varepsilon \bar{\beta}_{2}, \quad \beta_{3}=\varepsilon \bar{\beta}_{3}, \quad \beta_{4}=\varepsilon \bar{\beta}_{4} .
$$

We shaul deal with the oscillation in the resonance case, when there exists the following relation between the frequencies

$$
\omega=\frac{p}{q} \gamma+\varepsilon \delta
$$

$p, q$ are integers, $\delta$ is detuning. The partial solution of the equation (2.6) is found in the form of series

$$
y=a \cos \varphi+\varepsilon U_{1}(a, \psi, \theta)+\varepsilon^{2} U_{2}(a, \psi, \theta)+\varepsilon^{3}, \ldots,
$$

here $\varphi=\left(\frac{p}{q} \gamma t+\psi\right), \theta=\gamma t, a, \psi$ are the functions satisfying the following differential equations

$$
\begin{aligned}
& \frac{d a}{d t}=\varepsilon A_{1}(a, \psi)+\varepsilon^{2} A_{2}(a, \psi)+\varepsilon^{3}, \ldots \\
& \frac{d \psi}{d t}=\left(\omega-\frac{p}{q} \gamma\right)+\varepsilon B_{1}(a, \psi)+\varepsilon^{2} B_{2}(a, \psi)+\varepsilon^{3} \ldots
\end{aligned}
$$

It is easy to prove that the resonance occurs when

$$
\frac{p}{q}=\frac{1}{2} ; 1 ; 2 ; 3 .
$$

First of all, let's investigate the oscillation in the case

$$
\frac{p}{q}=\frac{1}{2}
$$

In the first approximation we have

$$
\begin{aligned}
y & =a \cos \varphi=a \cos \left(\frac{1}{2} \gamma t+\psi\right) \\
\gamma \frac{d a}{d t} & =a\left[h_{1} \alpha \gamma-P_{1} \cos 2 \psi\right] \\
a \gamma \frac{d \psi}{d t} & =a\left[\left(\omega^{2}-\frac{\gamma^{2}}{4}\right)-S_{1} a^{2}-2 h_{1} \omega^{2}-R_{1}+P_{1} \sin 2 \psi\right] .
\end{aligned}
$$


where

$$
h_{1}=\frac{\varepsilon \bar{\beta}_{2} Q_{0}}{4\left(\alpha^{2}+\gamma^{2}\right)}, \quad P_{1}=\frac{\varepsilon \bar{\beta}_{3} P_{0}}{4}, \quad S_{1}=\frac{3}{8} \varepsilon \bar{\beta}_{1}, \quad R_{1}=\frac{\varepsilon \bar{\beta}_{4} C_{0}}{2} .
$$

Stationary solution $a_{0}, \psi_{0}$ of the system of the equations (2.13) is determined from relations

$$
\begin{aligned}
& a_{0}\left(h_{1} \alpha \gamma-P_{1} \cos 2 \psi_{0}\right)=0, \\
& a_{0}\left[\left(\omega^{2}-\frac{\gamma^{2}}{4}\right)-S_{1} a_{0}^{2}-2 h_{1} \omega^{2}-R_{1}+P_{1} \sin 2 \psi_{0}\right]=0 .
\end{aligned}
$$

Eliminating the phase in (2.14), we get the equation of resonance curve for non-trivial stationary oscillation

$$
M\left(A_{0}^{2}, \eta^{2}\right)=\left[A_{0}^{2}+\frac{\eta^{2}}{4}+\left(2 h_{1}-1\right)+D\right]^{2}+B^{2} \alpha^{2} \eta^{2}-C^{2}=0 .
$$

From here we obtain

$$
A_{0}^{2}=\left(1-2 h_{1}\right)-\frac{\eta^{2}}{4}-D \pm \sqrt{C^{2}-B^{2} \alpha^{2} \eta^{2}}
$$

where

$$
A_{0}^{2}=\frac{S_{1} a_{0}^{2}}{\omega^{2}}, \quad \eta^{2}=\frac{\gamma^{2}}{\omega^{2}}, \quad C^{2}=\frac{P_{1}^{2}}{\omega^{4}}, \quad D=\frac{R_{1}}{\omega^{2}}, \quad B^{2}=\frac{h_{1}^{2}}{\omega^{2}} .
$$

To study the stability of the stationary oscillation, we set in (2.13) $a=a_{0}+\delta a, \psi=\psi_{0}+\delta \psi$, where $\delta a, \delta \psi$ are small pertubations. Substituting these expressions into equations (2.13) and neglecting the small quantities of high order, we receive the following variational equations

$$
\begin{aligned}
\gamma \frac{d \delta a}{d t} & =2 a_{0} P_{1} \sin 2 \psi_{0} \delta \psi, \\
a_{0} \gamma \frac{d \delta \psi}{d t} & =-2 a_{0}^{2} S_{1} \delta a+2 a_{0} P_{1} \cos 2 \psi_{0} \delta \psi,
\end{aligned}
$$

Using the Routh - Hurwi's criteria we get the following stability condition of stationary solution

$$
\begin{aligned}
& -2 a_{0} h_{1} \gamma^{2} \alpha>0 \\
& A_{0}^{2}+\frac{\eta^{2}}{4}+\left(2 h_{1}-1\right)+D>0
\end{aligned}
$$

The first inequality is always satisfied because $\alpha>0, h_{1}<0$. The second one will be realized, when the amplitude $A_{0}^{2}$ takes the values greater than $A^{2}$ lying on the backbone line corresponding to the equation

$$
A^{2}+\frac{\eta^{2}}{4}+\left(2 h_{1}-1\right)+D=0
$$

The relation (2.15) is plotted in Fig. 2 for the case:

$C^{2}=0.1, \quad B^{2}=0.05, \quad h_{1}=-0.025, \quad D=0.05$

$\alpha^{2}=0.6$ (curve 1); $\alpha^{2}=0.7$ (curve 2); $\alpha^{2}=0.8$ (curve 3).

The fat plots correspond to the stable state of the oscillation where the stability condition (2.19) is valid.

For the stationary solution $a_{0}=0$, the variational equations are of the form

$$
\begin{aligned}
\gamma \frac{d(\delta a)}{d t} & =\left(h_{1} \alpha \gamma-P_{1} \cos 2 \psi_{0}\right) \delta a \\
0 & =\left[\left(\omega^{2}-\frac{\gamma^{2}}{4}\right)-2 h_{1} \omega^{2}-R_{1}+P_{1} \sin 2 \psi_{0}\right] .
\end{aligned}
$$


The stability condition of this solution is

$$
\left[\frac{\eta^{2}}{4}+\left(2 h_{1}-1\right)+D\right]^{2}+d^{2} B^{2} \eta^{2}-C^{2}>0
$$

From the equation of resonance curve (2.15) when $A_{0}^{2}=0$, we have

$$
M\left(0, \eta^{2}\right)=\left[\frac{\eta^{2}}{4}+\left(2 h_{1}-1\right)+D\right]^{2}+\alpha^{2} B^{2} \eta^{2}-C^{2}
$$

If

$$
\frac{\left(1-2 h_{1}-D\right)-\sqrt{\left(1-2 h_{1}-D\right)^{2}-C^{2}}}{2 B^{2}}<\alpha^{2}<\frac{\left(1-2 h_{1}-D\right)+\sqrt{\left(1-2 h_{1}-D\right)^{2}-C^{2}}}{2 B^{2}},
$$

the equation (2.23) has not solution, the condition (2.22) always is realized. In this case, the resonance curve, expressed by the equation (2.16) will be upon or under the axis $\left(O \eta^{2}\right)$ and the stationary solution $\left(A_{0}^{2}=0\right)$ is always stability.

Now let's consider the parametric oscillation for the case $p / q=1$. In this case we have the averaging equation in the form

$$
\begin{aligned}
2 \gamma \frac{d a}{d t} & =a\left[h_{1} \alpha \gamma-\bar{\beta}_{4}\left(B_{2} \cos 2 \dot{\psi}+c_{2} \sin 2 \psi\right)\right] \\
2 a \gamma \frac{d \psi}{d t} & =a\left[\left(\omega^{2}-\gamma^{2}\right)-S_{1} a^{2}-h_{1} \omega^{2}-\bar{\beta}_{4}\left(C_{0}-B_{2} \sin 2 \psi+c_{2} \cos 2 \psi\right)\right] .
\end{aligned}
$$

where we denote

$$
h_{1}=\frac{\varepsilon \bar{\beta}_{2} Q_{0}}{2\left(\omega^{2}+d^{2}\right)}, \quad B_{2}=\frac{\varepsilon b_{2}}{4}, \quad C_{2}=\frac{\varepsilon c_{2}}{4}, \quad S_{1}=\frac{3}{8} \varepsilon \bar{\beta}_{1}, \quad C_{0}=\frac{\varepsilon c_{0}}{2} .
$$

From (2.21) we obtain the following equation for the amplitude of the nontrivial stationary oscillation

$$
A_{0}^{2}=\left(1-h_{1}\right)-\eta^{2}-D_{1} \pm \sqrt{C_{1}^{2}-B^{2} \alpha^{2} \eta^{2}}
$$

where

$$
A_{0}^{2}=\frac{S_{1} a_{0}^{2}}{\omega^{2}}, \quad \eta^{2}=\frac{\gamma^{2}}{\omega^{2}}, \quad C_{1}^{2}=\frac{\bar{\beta}_{4}^{2}\left(B_{2}^{2}+C_{2}^{2}\right)}{\omega^{2}}, \quad D_{1}=\bar{\beta}_{4} C_{0}, \quad B_{1}^{2}=\frac{h_{1}^{2}}{\omega^{2}} .
$$

It is seen that the amplitude $A_{0}^{2}$ decreases and the stability region of the stationary oscillation is also narrow in comparison with the case $q=1 / 2[4]$.

\section{CONCLUSION}

1. Where taking into account the lengthener $\varepsilon_{0}$ of the shaft's axis in the system investigated there exist three resonance cases, they are not observed for system having linear geometric character. The ultraharmonic $(p / q=1 / 2)$ oscillations are not studied, however, it may be seen that their amplitude and the stability zone become less in comparision with the examined case.

2. From the figures presented, we can see that the nonlinear hereditary of material decreases the amplitude of the parametric oscillation, that can be disappeared when value of $\alpha^{2}$ is sufficiently great.

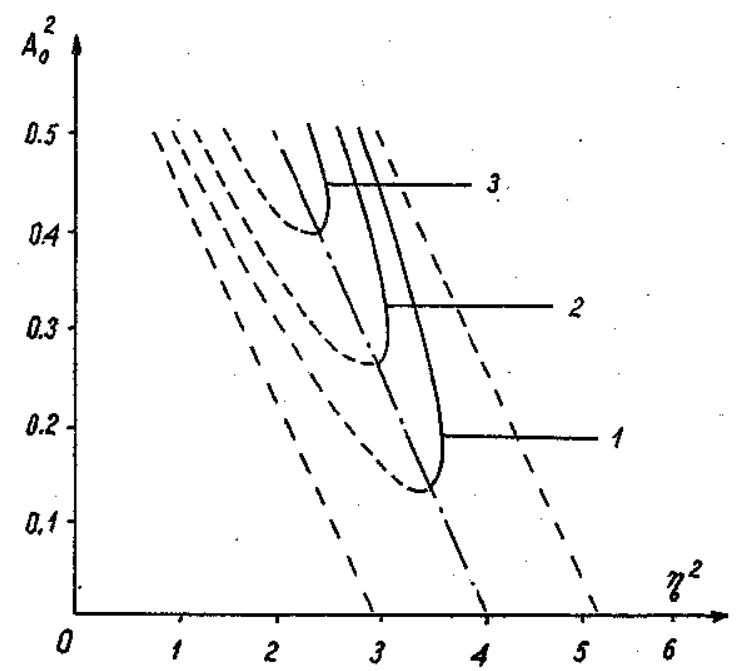

Fig. 2 
3. I wish to express here my gratitude and warmest thanks to Prof. Dr. Nguyen Van Dao for his keen interest in this work completed.

This publication is completed with financial support from the National Basic Research Program in Natural Sciences.

\section{REFERENCES}

1. Osinski Z., Nguyen Van Dao. Parametric oscillation of an uniform beam in a theological model. Proceeding of the second National Conference on Mechanics, Hanoi 1977.

2. Nguyen Van Dao. Nonlinear oscillations of high order systems. National Center for Scientific Research of Vietnam, Hanoi 1972.

3. Hoang Van Da, Nguyen Van Dao. Beitrag zur-untursuchung der nichtlinearen schwingungen mechanischen systems mit elastischplastichen materialeingschafften. TH. Otto von Guericke. Technische Mechanik. DDR, No 1, 1986.

4. Hoang Van Da. Parametric vibration of a prismatic shaft with nonlinear heredity. Stroinicky Casopis. Roc 35, Cislo 5, CSSR p. 582.

5. Hoang Van Da. Parametric oscillation of the shaft making mention of creep of material and nonlinear boundary conditions. Journal of applied Mechanics, Roumanie, Bucarest. T. 29, No 5,1934 , p. $463-474$.

6. Hoang Van Da. Construction of the asymptotic solutions of the high order partial differential equation with two space variable. Journal of Mathematics, Ukrainian Academy of sciences, Kiev, T. 36, No 1, 1984, p. 87-93.

7. Hoang Van Da. Parametric oscillation of a rectangular thin viscous elastic plate. Journal of applied Mechanics, Ukrainian Academy of sciences, Kiev, T.19, No 12, 1983, p. 120-124.

8. Hoang Van Da. Parametric excited oscillation of the shaft with nonlinear elastic heredity. Journal of applied Mechanics. Ucrainian Academy of Sciences, Kiev, T. 21, No 6, 1985, p. 85-88.

9. Hoang Van Da. Asymptotic solutions of the high order partial differential equation with two space variables and nonlinear conditions. Journal of Mathematics, Ukrainian Academy of sciences, Kiev, T. 32, No 4, 1986, p. 527-531.

10. Mitropolsky Yu. A., Berezovsky A. A., Turgunov N. Parametric forced oscillations of the shaft with nonlinear elastic. Journal of Mathematics, Ukrainian Akademy of sciences, 1975, No 3, p. 395-400.

11. Smidt G. Parametric oscillations, Moscow, 1978.

12. Nguyen Xuan Hung. Parametric vibration of the visco-elstic shaft with the nonlinear elastic law and the nonlinear inside viscidity. Journal of Applied Mechanics. Ukrainian Academy of science, 1979, T. XV, No 9.

Received June 20, 1994

\section{DAO ĐộNG THÔNG SỐ CƯA DẦM LA̛NG TRỤ KỂ ĐẾN TÍNH DI TRUYỀN VA PHI TUYẾN HINNH HỌC}

Trong bài báo này, tác giả nghiên cứu dao động thông số của dầm lăng trụ có kể đến tính di truyền cuia vật liệu và biến dạng dài $\varepsilon_{0}$ của trục đối xứng của dầm.

Kết quả cho thấy rằng xuất hiện thêm ba chế độ cộng hướng $p / q=1,2,3$ mà trước đó chưa được xem xét.

Khi $p / q=1 / 2$ đường cong cộng hương đã được xây dụng, sự ồn định của nghiệm dừng đã được khăo sát và có thể chọn $\alpha^{2}$ đủ lớn thì dao động thông số. biến mất. 